\title{
Central Pancreatectomy for Central Pancreatic Lesions: A Single-Institution Experience
}

\author{
Senthil Kumar $\mathrm{P}^{1}$, Sakthivel Harikrishnan ${ }^{1}$, Jeswanth Satyanesan ${ }^{1}$ \\ 1. Surgical Gastroenterology and Liver Transplant, Government Stanley Medical College, Chennai, IND
}

Corresponding author: Sakthivel Harikrishnan, shakthivel.hsurgeon@gmail.com

\section{Abstract \\ Background}

Pancreaticoduodenectomy and distal pancreatectomy are radical procedures for pancreatic lesions with high postoperative morbidity and mortality even in experienced hands. Central pancreatectomy is an alternative less radical procedure for centrally located pancreatic lesions that are benign or have a low malignant potential. It involves removing the central portion of the pancreas and has the advantage of preserving the pancreatic parenchyma, thereby decreasing the postoperative endocrine and exocrine insufficiencies.

\section{Methods}

We conducted a prospective study of six cases of central pancreatectomy in the Department of Surgical Gastroenterology and Liver Transplant, Government Stanley Medical College, India, between the years 2015 and 2019. All patients with lesions in the neck and proximal body of the pancreas were clinically and radiologically evaluated, and those with benign or borderline malignant lesions underwent central pancreatectomy by a standardized technique.

\section{Results}

The mean age of the patients was 27.8 years (range: 14 years - 37 years). Most of the patients were females (66.6\%). The most common presenting symptom was abdominal pain, and the most common diagnosis was solid pseudopapillary neoplasm (83.3\%). The mean diameter of the lesion was $6.1 \mathrm{~cm}$. All patients underwent pancreaticojejunostomy of the distal stump. The median operative time and the blood loss were 310 minutes and $85 \mathrm{ml}$, respectively. Two patients developed biochemical postoperative pancreatic fistula, and in the long-term follow-up, none of them developed endocrine or exocrine insufficiency.

\section{Conclusion}

Central pancreatectomy is a safe and effective alternative for benign and low-grade lesions in the neck and body of the pancreas in which the head of the pancreas and a significant portion of the distal body and tail of the pancreas is uninvolved. Standardization of this pancreas-preserving procedure will result in better outcomes.

Review began 06/17/2021 Review ended 06/24/2021 Published 07/02/2021

(c) Copyright 2021

$P$ et al. This is an open access article distributed under the terms of the Creative Commons Attribution License CC-BY 4.0., which permits unrestricted use, distribution, and reproduction in any medium, provided the original author and source are credited.
Categories: Gastroenterology, General Surgery

Keywords: central pancreatectomy, pancreas preserving resection, solid pseudopapillary neoplasm, cp, postoperative pancreatic fistula

\section{Introduction}

Traditional surgeries for pancreatic lesions include pancreaticoduodenectomy (PD) and distal pancreatectomy with or without splenectomy. Even in experienced hands, these surgeries carry a significant morbidity and mortality risk. These risks can be accepted if an oncological cure is the primary intent. Pancreas-preserving resections are surgeries that are aimed at preserving pancreatic parenchyma, thereby decreasing the surgical complications and the postoperative endocrine and exocrine insufficiencies in the long term. Pancreas-preserving surgeries include duodenum-sparing head resection, enucleation, and central pancreatectomy (CP) [1]. CP has become the standard surgical procedure for benign and borderline lesions in the body and neck of the pancreas. In this prospective study, we report our experience with six cases of central pancreatectomy done for benign lesions in the neck and proximal body of the pancreas.

\section{Materials And Methods}

Patients and methods

From January 2015 to December 2019, six cases underwent central pancreatectomy in the Department of Surgical Gastroenterology, Government Stanley Medical College, Chennai, India. All patients underwent routine blood investigations, including tumor marker (CA19-9) and imaging by pancreatic protocol CT and MRI (Figure 1). 


\section{Cureus}

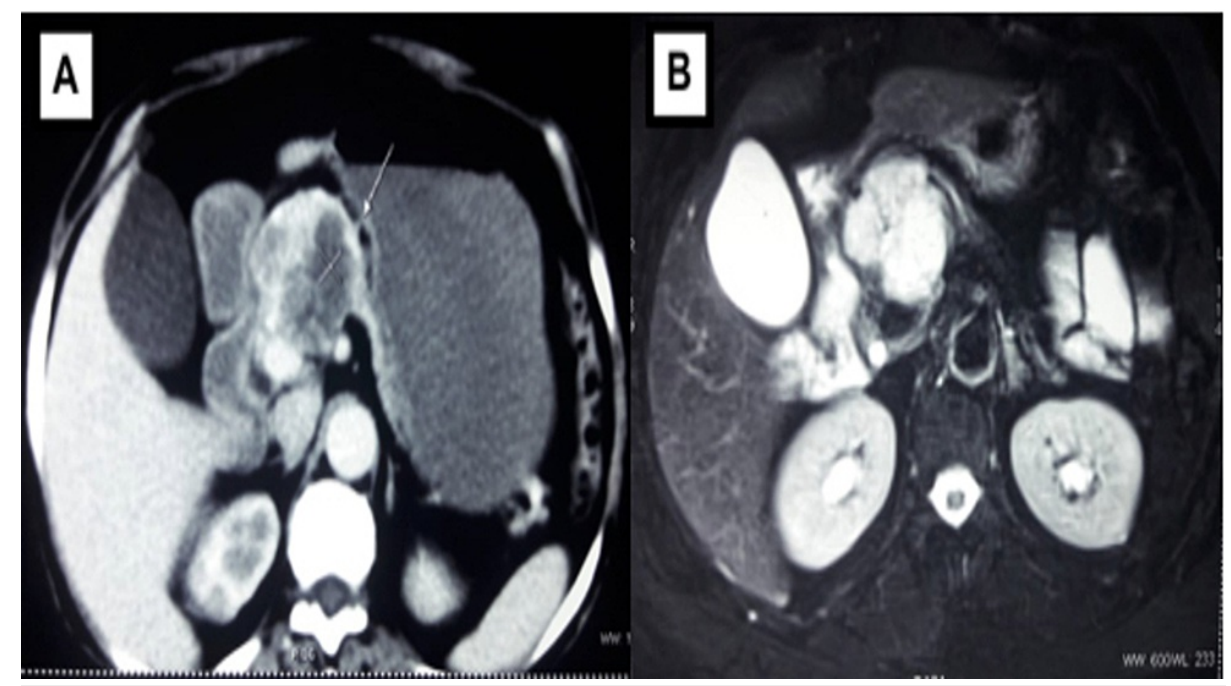

FIGURE 1: A \& B: CT and MRI picture showing a heterogeneously enhancing cystic lesion in the neck of the pancreas

We assessed the location and characteristics of the tumor, the relationship with the mesenteric vessels, and the remnant pancreatic volume in the imaging. Patients who had a suspicious benign lesion to the left of the gastroduodenal artery and close to the splenomesenteric confluence, with adequate remnant volume, were taken as ideal candidates for central pancreatectomy. Endoscopic ultrasound (EUS) was not included in our protocol to evaluate the cystic lesions in the pancreas due to nonavailability. Central pancreatectomy was done by the standardized technique described below.

\section{Surgical technique}

The abdomen was opened by a Makuuchi incision. The lesser sac was entered after dividing the gastrocolic ligament and preserving the gastroepiploic vessels. The adhesions between the posterior surface of the stomach and the pancreas were divided, and the anterior surface of the pancreas was exposed. The inferior border of the pancreas was mobilized at the level of the neck of the pancreas, and a tunnel was created between the posterior aspect of the neck of the pancreas and the portal vein. An umbilical tape was passed through this tunnel and looped around the neck of the pancreas as proximal control (Figure $2 \mathrm{~A}$ ). The splenic artery is preserved at the superior border of the pancreas. After mobilizing the inferior border of the pancreas and carefully preserving the splenic vein, distal control is taken by passing an umbilical tape $1 \mathrm{~cm}$ away from the lesion in the body of the pancreas (Figure $2 B$ ). The margins are marked on the pancreatic parenchyma 5 to $10 \mathrm{~mm}$ away from the lesion proximally and distally. Pancreatic parenchyma was transected with diathermy proximal and distal to the lesion with adequate precautions not to injure the splenoportal axis (Figure 2C). The pancreatic duct was identified in the proximal and distal cut surfaces and cannulated with an infant feeding tube (Figure 2D). 


\section{Cureus}

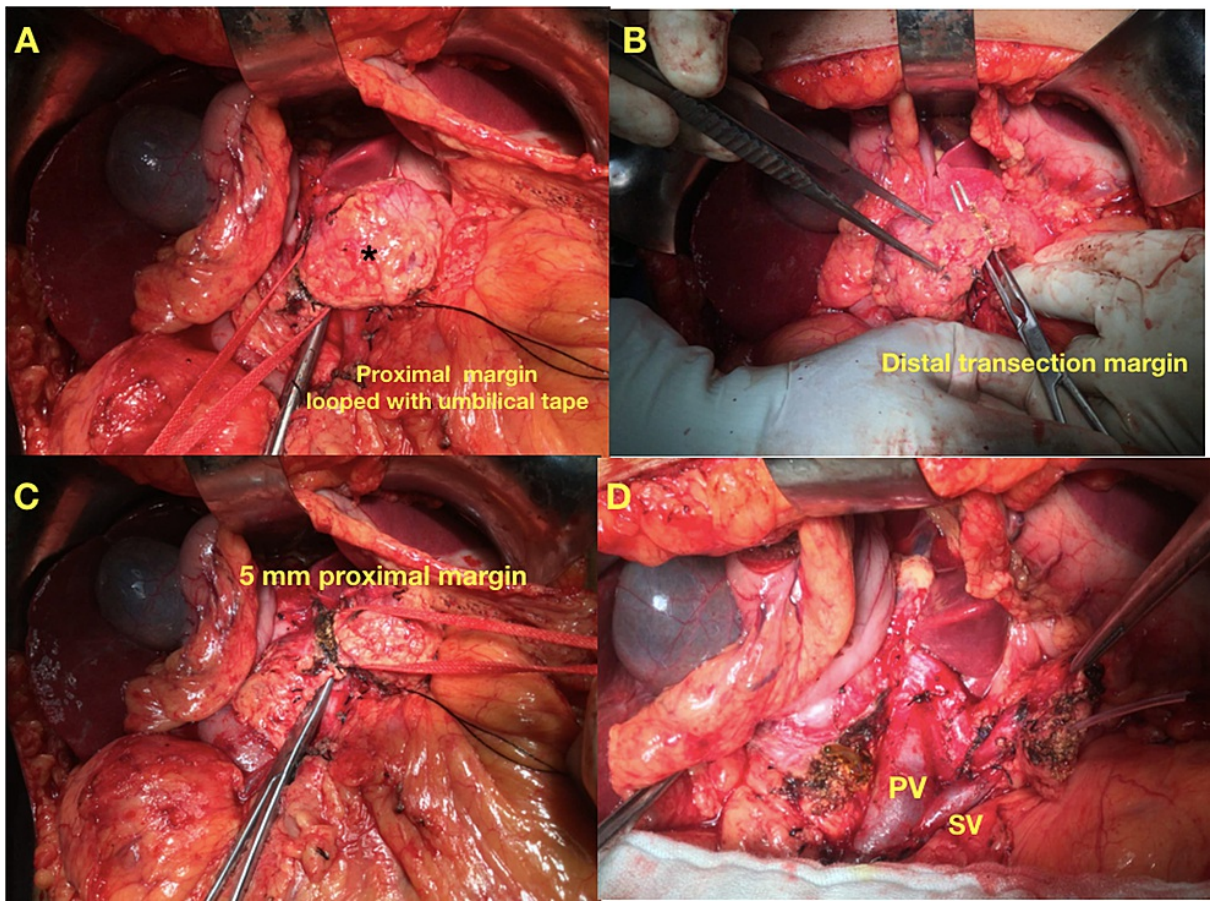

FIGURE 2: A: Lesion (*) in the proximal body of the pancreas, and after tunneling, an umbilical tape is looped around the pancreas. B: Pancreas distal to the lesion is mobilized, and an instrument is passed between the splenic vein and the posterior surface of the pancreas for distal transection. C: Proximal pancreatic parenchymal transection with $5 \mathrm{~mm}$ of normal pancreatic parenchyma proximal to the lesion. D: Postresection picture showing the splenoportal axis and infant feeding tube inserted into the proximal and distal transected pancreatic duct

After securely closing the proximal pancreatic duct with 3-0 Prolene sutures, horizontal mattress sutures were taken on the proximal transected pancreatic surface. The distal pancreas was mobilized from the splenic vein posteriorly for $3 \mathrm{~cm}$. Pancreaticojejunostomy was done end to side with the loop of jejunum passed through the retrocolic window using the Blumgart technique. Drains were placed in the lesser sac, and the Morrisons pouch and abdomen were closed in layers. At the one-year follow-up, the endocrine function was assessed by measuring fasting and postprandial blood sugars. Patients who had weight loss, diarrhea, and fatty stools were considered to have exocrine insufficiency.

\section{Results}

A total of six patients underwent a central pancreatectomy. The mean patient age was 27.8 years (range: 14 years - 37 years) and most of them are females (66.6\%). The most common presenting symptom was abdominal pain. All patients had normal CA19-9 levels. One patient was referred to us after EUS biopsy showed evidence of a solid pseudopapillary neoplasm. The most common indication was a solid pseudopapillary neoplasm (83.3\%) followed by mucinous cystadenoma (16.7\%). The mean tumor size was 6.1 $\mathrm{cm}$. All patients underwent pancreaticojejunostomy. The median operative time and blood loss were 310 minutes and $85 \mathrm{ml}$, respectively. Two patients (33\%) developed a biochemical postoperative pancreatic fistula. The mean duration of hospital stay was 12 days. During the one-year follow-up, none of the patients developed impaired glucose tolerance or pancreatic insufficiency. The clinical characteristics of the patients are summarized in Table 1. 
series underwent a standardized end-to-side pancreaticojejunal anastomosis and didn't have any clinically relevant POPF, and in the one-year follow-up, none of them had exocrine or endocrine insufficiency.

The following are the main advantages of central pancreatectomy over the standard pancreatic resections based on the available literature: 1 . Whipple's procedure and distal pancreatectomy is associated with endocrine insufficiencies in $15 \%$ and $50 \%-60 \%$ of the patients, respectively. A meta-analysis has shown that central pancreatectomy significantly reduces the risk of the onset of diabetes when compared to distal pancreatectomy (5.5\% vs. 23.6\%). The preservation of exocrine function was borderline significant when compared to the distal pancreatectomy [11]. 2. The gastrointestinal and biliary continuity is unaltered, thereby avoiding metabolic and nutritional complications. 3 . The chances of preserving the spleen are more when compared to distal pancreatectomy. 4. Though the occurrence of the pancreatic fistula is high after central pancreatectomy, most of them are biochemical leaks or grade B fistulas, which can be managed conservatively or by percutaneous drainage [11-12].

Central pancreatectomy can also be done by the laparoscopic approach and robotic-assisted laparoscopic approach with comparable fistula rates to open pancreaticogastrostomy is the preferred reconstruction technique after the laparoscopic approach because of the anatomic proximity and technical ease [13-15]. A large case series of 100 patients who underwent central pancreatectomy showed that the learning curve improved the operative time and the blood loss but the clinically relevant POPF rates (32\%) and the biochemical leak rate (14\%) hasn’t shown much improvement [16]. Both these approaches reduce postoperative morbidity when compared with the open procedure.

Distal pancreatectomy (DP) is the other most commonly considered surgical option for lesions in the neck and the body of the pancreas. Several studies have compared CP vs. DP as a surgical option for benign lesions in the proximal body of the pancreas. The study by Ocuin et al. showed that the central pancreatectomy patients had a significantly higher risk of complications than extended left pancreatectomy. There was no significant risk of major complications or hospital stay in the CP group. Patients who had undergone extended left pancreatectomy had a higher incidence of clinically significant new-onset diabetes [17]. The meta-analysis of Iacono et al. comparing central pancreatectomy with distal pancreatectomy concluded that though the risk of overall morbidity and fistula was high, the risk of reoperation and longterm endocrine failure showed a clinically significant reduction [11]. A multicenter study in which our center took part in India on cystic tumors of the pancreas reported that the most common cystic tumor was solid pseudopapillary epithelial neoplasm (28\%) and central pancreatectomy was done in only $8.5 \%$ of the cases [18]. In our center, we select patients with definitely benign lesions in the body of the pancreas based on imaging, clinical parameters, and intraoperatively if sufficient remnant pancreatic head volume and tail volume is present, we proceed with CP. Patients who have insufficient pancreatic tail volume undergo distal pancreatectomy.

The main limitation of the study is the small sample size because of the rare occurrence of benign lesions in the body of the pancreas suitable for central pancreatectomy. There are no randomized trials to compare the parenchyma-preserving $\mathrm{CP}$ with radical pancreatic resections like distal pancreatectomy or pancreaticoduodenectomy. The published literature includes mostly large case series, which clearly shows better long-term endocrine and exocrine functions with CP. Since we didn't take a preoperative biopsy to objectively rule out a malignant lesion, central pancreatectomy would be oncologically inappropriate if the lesion turns out to be malignant. The other drawback is, we didn't objectively measure the pancreatic exocrine insufficiency in follow-up. Patients who had steatorrhoea and significant weight loss were considered to have exocrine insufficiency in our series.

\section{Conclusions}

Central pancreatectomy is a safe and effective alternative for benign and low-grade lesions in the neck and the proximal body of the pancreas in which the head of the pancreas and a significant portion of the distal body and tail of the pancreas is uninvolved. Theoretically, though the risk of the pancreatic fistula is high, it is usually easily manageable. Standardization of this surgical procedure might lead to better outcomes and quality of life in the long term.

\section{Additional Information \\ Disclosures}

Human subjects: Consent was obtained or waived by all participants in this study. Government Stanley Medical College issued approval NA. Ethics approval and IRB number were not obtained, as it is a small case series of only six cases; instead, informed consent was obtained from all the patients. Animal subjects: All authors have confirmed that this study did not involve animal subjects or tissue. Conflicts of interest: In compliance with the ICMJE uniform disclosure form, all authors declare the following: Payment/services info: All authors have declared that no financial support was received from any organization for the submitted work. Financial relationships: All authors have declared that they have no financial relationships at present or within the previous three years with any organizations that might have an interest in the submitted work. Other relationships: All authors have declared that there are no other 


\section{References}

1. Brown KM, Shoup M, Abodeely A, Hodul P, Brems JJ, Aranha GV: Central pancreatectomy for benign pancreatic lesions. HPB (Oxford). 2006, 8:142-7. 10.1080/13651820510037611

2. Celis J, Berrospi F, Ruiz E, Payet E, Luque C: Central pancreatectomy for tumors of the neck and body of the pancreas. J Surg Oncol. 2001, 77:132-5. 10.1002/jso.1083

3. Ikeda S, Matsumoto S, Maeshiro K, Miyazaki R, Okamoto K, Yasunami Y: Segmental pancreatectomy for the diagnosis and treatment of small lesions in the neck or body of the pancreas. Hepatogastroenterology. 1995, 42:730-3.

4. Warshaw AL, Rattner DW, Fernández-del Castillo C, Z'graggen K: Middle segment pancreatectomy. A novel technique for conserving pancreatic tissue. Arch Surg. 1998, 133:327-31. 10.1001/archsurg.133.3.327

5. Xiao W, Zhu J, Peng L, Hong L, Sun G, Li Y: The role of central pancreatectomy in pancreatic surgery: a systematic review and meta-analysis. HPB (Oxford). 2018, 20:896-904. 10.1016/j.hpb.2018.05.001

6. Roggin KK, Rudloff U, Blumgart LH, Brennan MF: Central pancreatectomy revisited. J Gastrointest Surg. 2006, 10:804-12. 10.1016/j.gassur.2005.11.012

7. Paiella S, De Pastena M, Faustini F, et al.: Central pancreatectomy for benign or low-grade malignant pancreatic lesions - a single-center retrospective analysis of 116 cases. Eur J Surg Oncol. 2019, 45:788-92. 10.1016/j.ejso.2018.11.021

8. Lee D-H, Han Y, Byun Y, Kim H, Kwon W, Jang J-Y: Central pancreatectomy versus distal pancreatectomy and pancreaticoduodenectomy for benign and low-grade malignant neoplasms: a retrospective and propensity score-matched study with long-term functional outcomes and pancreas volumetry. Ann Surg Oncol. 2020, 27:1215-24. 10.1245/s10434-019-08095-Z

9. Hirono S, Tani M, Kawai M, et al.: A central pancreatectomy for benign or low-grade malignant neoplasms . J Gastrointest Surg. 2009, 13:1659-65. 10.1007/s11605-009-0934-3

10. Allendorf JD, Schrope BA, Lauerman MH, Inabnet WB, Chabot JA: Postoperative glycemic control after central pancreatectomy for mid-gland lesions. World J Surg. 2007, 31:164-8; discussion 169-70. 10.1007/s00268-005-0382-5

11. Iacono C, Verlato G, Ruzzenente A, et al.: Systematic review of central pancreatectomy and meta-analysis of central versus distal pancreatectomy. Br J Surg. 2013, 100:873-85. 10.1002/bjs.9136

12. Zhou Y-M, Zhang X-F, Wu L-P, Su X, Li B, Shi L-H: Pancreatic fistula after central pancreatectomy: case series and review of the literature. HBPD INT. 2014, 13:203-8. 10.1016/s1499-3872(14)60032-1

13. Sa Cunha A, Rault A, Beau C, Collet D, Masson B: Laparoscopic central pancreatectomy: single institution experience of 6 patients. Surgery. 2007, 142:405-9. 10.1016/j.surg.2007.01.035

14. Hamad A, Novak S, Hogg ME: Robotic central pancreatectomy. J Vis Surg. 2017, 3:94. 10.21037/jovs.2017.05.13

15. Dokmak S, Aussilhou B, Samir Ftériche F, Soubrane O, Sauvanet A: Laparoscopic central pancreatectomy: surgical technique. J Visc Surg. 2020, 157:249-53. 10.1016/j.jviscsurg.2020.04.009

16. Shi Y, Wang Y, Wang J, et al.: Learning curve of robot-assisted middle pancreatectomy (RMP): experience of the first 100 cases from a high-volume pancreatic center in China. Surg Endosc. 2020, 34:3513-20. 10.1007/s00464-019-07133-x

17. Ocuin LM, Sarmiento JM, Staley CA, Galloway JR, Johnson CD, Wood WC, Kooby DA: Comparison of central and extended left pancreatectomy for lesions of the pancreatic neck. Ann Surg Oncol. 2008, 15:2096-103. 10.1245/s10434-008-9987-x

18. Chaudhari VA, Pradeep R, Ramesh H, et al.: Surgery for cystic tumors of pancreas: report of high-volume, multicenter Indian experience over a decade. Surgery. 2019, 166:1011-6. 10.1016/j.surg.2019.07.013 